\title{
NATIONAL PARK SERVICE AREAS COOPERATING WITH \\ U.W.-N.P.S. RESEARCH CENTER
}

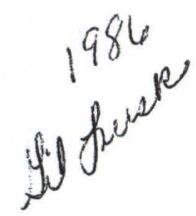

1. GLACIER NATIONAL PARK West Glacier, MT 59936 Superintendent: Telephone: (406) 888-5411 $1,013,598.42$ Acres Fed-1,012,599.10; Nonfed-999.32

15. YELLOWSTONE NATIONAL PARK Yellowstone Park, WY 82190

Superintendent: Robert Barbee Telephone: (307) 344-7381 $2,219,822.70$ Acres Fed-2,219,736.88; Nonfed-85.82

10. DEVILS TOWER NATIONAL MONUMENT

Devils Tower, WY 82714

Superintendent: Homer $\AA$. P te Robinson

Telephone: (307) 467-5370 1,346.91 Acres, Federal

11. FORT LARAMIE NATIONAL HISTO RIC STTE

Fort Laramie, WY 82212 Superintendent: Gary K. Howe Telephone: (307) 837-2221 571.36 Acres Fed-562.80; Nonfed-8.56

12. FOSSII BUTTE NATIONAL MONUMENT

P. O. BoX 527

Kemmerer, WY 83101

Superintendent: Paul Guraedy

Telephone: (307) 877-3450

8,178 Acres

Fed-7,417.64; Nonfed-760.36

16. BIGHORN CANYON NATIONAL RECREATION AREA

P. O. Box 458

Fort Smith, MT 59035

Superintendent: William G. Binnewies Telephone: (406) 666-2412 $120,157.88$ Acres

Fed-65,608.16; Nonfed-54,549.72

17. CUSTER BATTLEFIELD NATIONAL MONUMENT

P. O. Box 39

Crow Agency, MT 59022

Superintendent: James-Court

Telephone: (406) 638-2622

765.34 Acres

18. BIG HOLE NATIONAL BATTLEFIELD

P. O. Box 237

Wisdom, MT 59761

Superintendent: Alfred Schulmeyer 655.61 Acres, Federal

13. GRAND TETON NATIONAL PARK 19. GRANT-KOHRS RANCH

P. O. Drawer 170

Moose, WY 83012

NATIONAL HISTORIC STTE

Superintendent: Jack Stark

Telephone: (307) 733-2880

$310,417.98$ Acres

Fed-305,504.53; Nonfed-4,913.45

P. O. Box 790

Deer Lodge, MT 59722

Superintendent: Jimmy D. Taylor

Telephone: (406) 846-2070

$1,527.90$ Acres

Fed-1,322.48; Nonfed-205.42

14. JOHN D. ROCKEFELLER JUNIOR, MEMORIAL PARKWAY

\& Grand Teton National Park

P. O. Drawer 170

Moose, WY 83012

Telephone: (307) 733-2880

23,777.22 Acres, Federal 


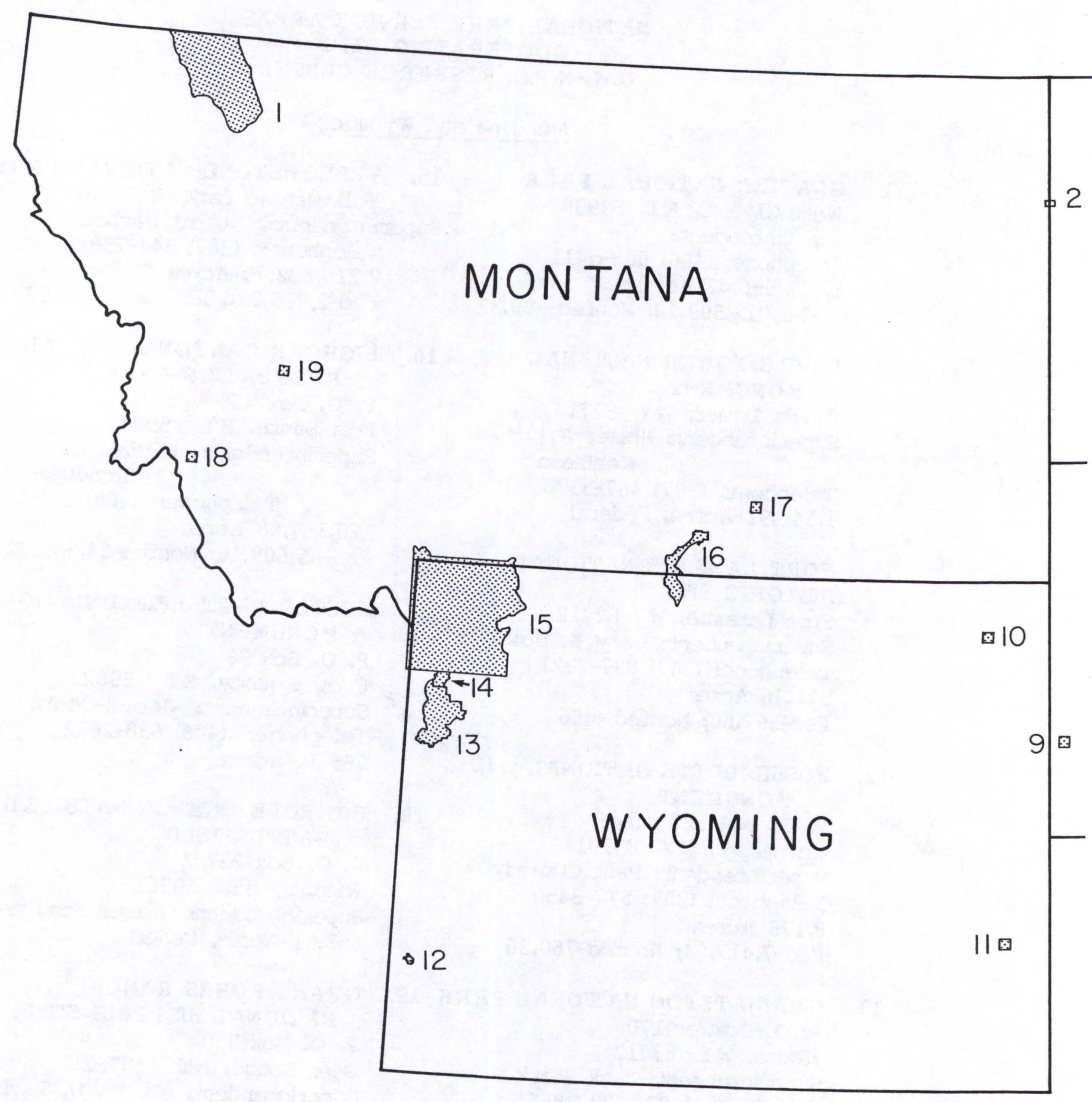




\section{NATIONAL PARK SERVICE AREAS \\ COOPERATING WITH \\ U.W.-N.P.S RESEARCH CENTER}

\section{North Dakota and South Dakota}

2. FORT UNION TRADING POST

NATIONAL HISTORIC SITE

Buford Route

Williston, ND 58801

Area Manager: Paul Hedren

Telephone: (701) 572-9083

398.45 Acres

Fed-392.03; Nonfed-6.42

3. THEODORE ROOSEVELT

NATIONAL PARK

Medora, ND 58645

Superintendent: Harvey D.

Wickware

Telephone: (701) 623-4466

$70,408.64$ Acres

Fed-69,675.88; Nonfed-732.76

4. KNIFE RIVER INDIAN VILLAGES

NATIONAL HISTORIC SITE

P. O. Box 175

Stanton, ND 58571

Area Manager: Michael Holm

Telephone: (701) 795-33099.

1,309.51 Acres

Fed-170.08; Nonfed-1,139.43

5. INTERNATIONAL PEACE GARDENS

P. O. Box 419

Dunseith, ND 58637

Telephone: (701) 623-4466
6. BADLANDS NATIONAL MONUMENT

P. O. Box 6

Interior, SD 57750

Superintendent: Don Falvey

Telephone: (605) 433-5361

243,302.23 Acres

Fed-152,289.56; Nonfed-91,012.77

7. WIND CAVE NATIONAL PARK

Hot Springs, SD 57747

Superintendent: Ernest Ortega

Telephone: (605) 727-2301

28,060.03 Acres, Federal

8. MOUNT RUSHMORE NATIONAL MEMORIAL

Keystone, SD 57751

Superintendent: Dan Wenk

Telephone: (605) 574-2523

1,278.45 Acres

Fed-1,245.95; Nonfed-32.50

9. JEWEL CAVE NATIONAL

MONUMENT

Custer, SD 57730

Management Assistant: Bennis $\mathrm{I}$. Ditmanson

Telephone: (605) 673-2288

1,274.56 Acres, Federal 


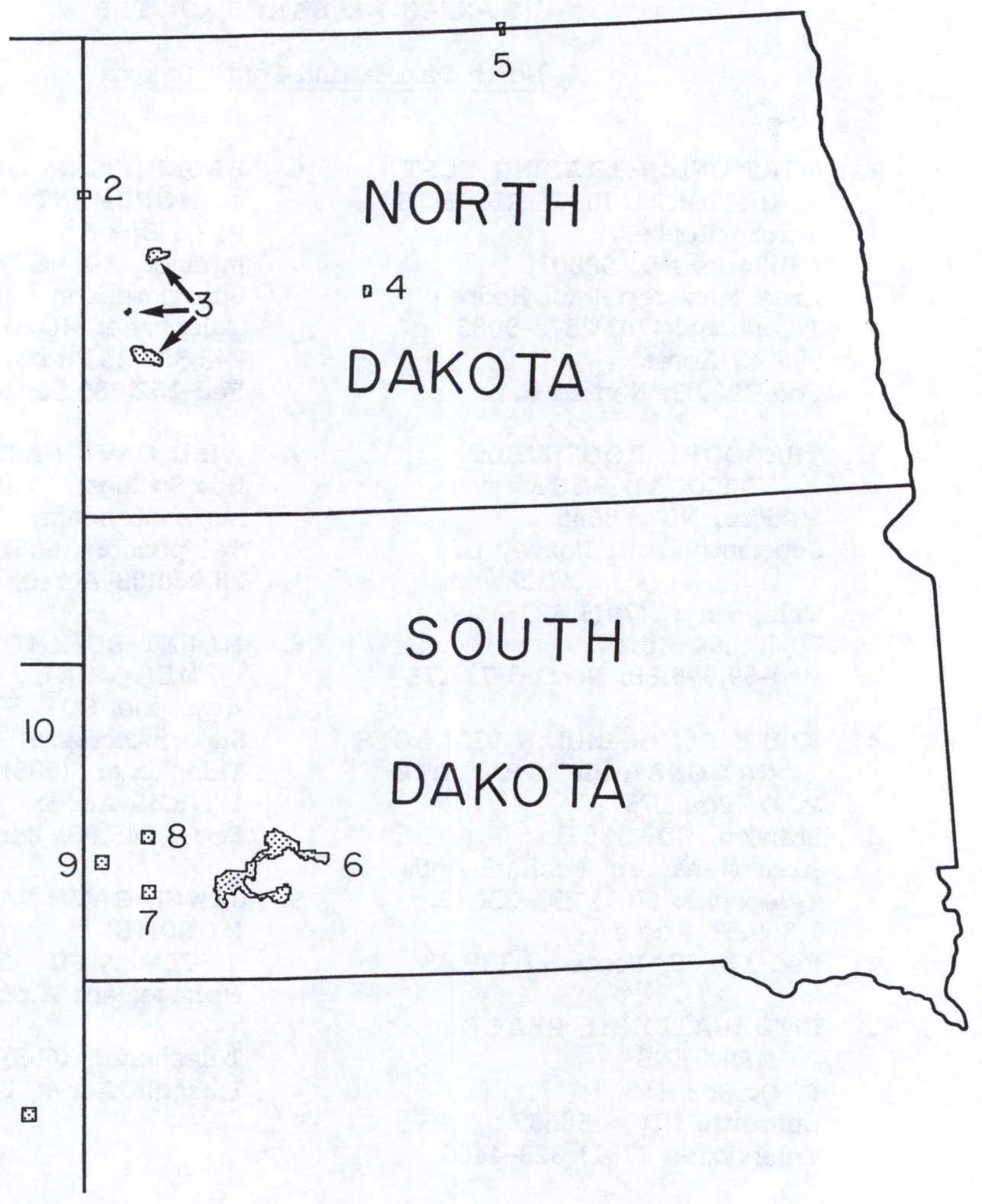




\section{NATIONAL PARK SERVICE AREAS COOPERATING WITH \\ U.W.-N.P.S. RESEARCH CENTER}

\section{Utah}

20. CEDAR BREAKS NATIONAL MENUMENT

P. O. Box 749

Cedar City, UT 84720-0749

Superintendent: Elay Ałderson

Telephone: (801) 586-9451

6,154.60 Acres, Federal

21. GOLDEN SPIKE NATIONAL HISTO RIC SITE

P. O. Box 394

Brigham City, UT 84302-1099

Superintendent: Dennis Davies

2,203.20 Acres, Federal

22. GLEN CANYON NATIONAL RECREATION AREA

P. O. Box 1507

Page, AZ 86040-1507

Superintendent: John Lancaster

Telephone: (602) 765-7500

$1,236,880$ Acres

Fed-1,157,463; Nonfed-79,417

CANYONLANDS COMPLEX

446 South Main Street

Moab, UT 84532-2995

Superintendent: Peter Panry

Telephone: (801) 259-7164

23. ARCHES NATIONAL PARK

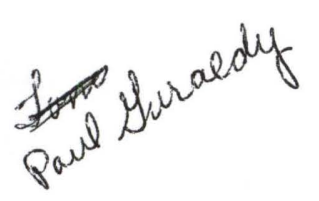

Unit Manager: Sherma-Bierhaus

Telephone: (801) 259-8161

$73,378.98$ Acres

Fed-65,097.59; Nonfed-8,281.39

24. CANYONLANDS NATIONAL PARK

Unit Manager: Peter Parry

Telephone: (801) 259-7164

337,570.43 Acres, Federal

25. NATURAL BRIDGES NATIONAL MONUMENT

Unit Manager: Gary Hasty

Telephone: (801) 557-1190

7,779.14 Acres, Federal
26. ZION NATIONAL PARK

Springdale, UT 84767-1099

Superintendent: Harry Grafe

Telephone: (801) 772-3256

$146,546.97$ Acres

Fed-142,461.82; Nonfed-4,085.15

27. CAPITOL REEF NATIONAL PARK Torrey, UT 84775-0001

Superintendent: Bob Reynolds

Telephone: (801) 425-3871

$241,865.48$ Acres

Fed-221,896.66; Nonfed-19,968.82

28. PIPE SPRING NATIONAL MONUMENT

Moccasin, A.Z 86022-1099

Superintendent: William Herr

Telephone: (602) 643-7105

29. DINOSAUR NATIONAL MONUMENT

P. O. Box 210

Dinosaur, CO 81610

Superintendent: Joe-Kennedy

Telephone: (303) 374-2216

$211,053.45$ Acres

Fed-203,629.17; Nonfed-7,424.28

30. TIMPANOGOS CAVE NATIONAL MONUMENT

Rt. 2, Box 200

American Fork, UT 84003

Superintendent: William Welman

Telephone: (801) 756-5238

250 Acres, Federal

31. BRYCE CANYON National

PARK

Bryce Canyon, UT 84717-0001

Superintendent: Sandra H. Key

Telephone: (801) 834-5322

35,835.08 Acres

Fed-35,832.58; Nonfed-2.50 


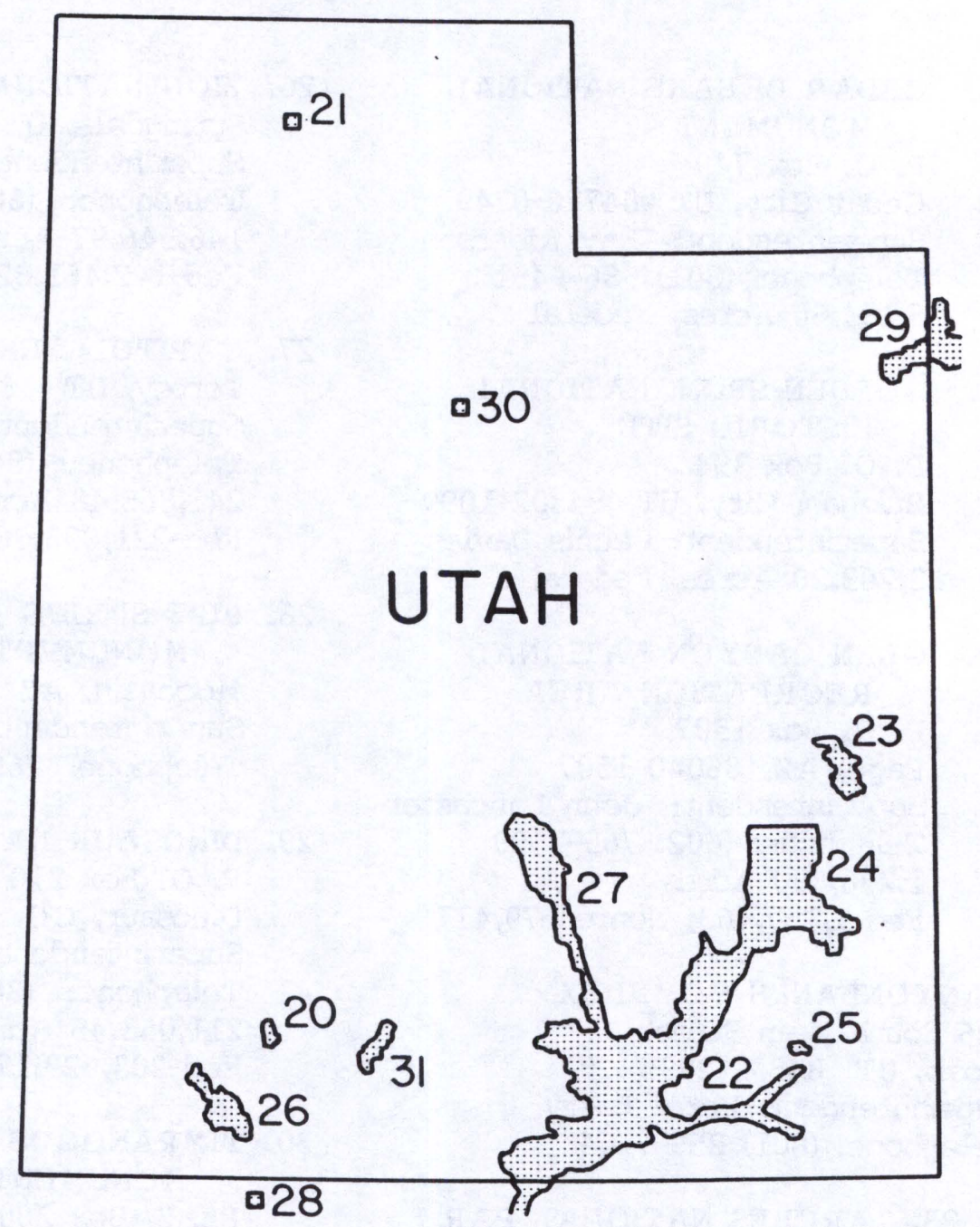

